\title{
Does Speech Rhythm Sensitivity Predict Children's Reading Ability 1 Year Later?
}

\author{
Andrew J. Holliman and Clare Wood \\ Coventry University
}

\author{
Kieron Sheehy \\ Open University
}

\begin{abstract}
There is a growing literature demonstrating that speech rhythm sensitivity is related to children's reading development, independent of phonological awareness. However, the precise nature of this relationship is less well understood, and further research is warranted to investigate whether speech rhythm sensitivity predicts the different components of reading over time. In this 1-year longitudinal study, 69 five- to 8-year-old English-speaking children completed a speech rhythm assessment at Time 1 along with other cognitive assessments and then completed a variety of reading assessments at Time 2 ( 1 year later). A series of hierarchical regression analyses revealed that after controlling for individual differences in age, vocabulary, and phonological awareness, speech rhythm sensitivity was able to predict unique variance in word reading and the phrasing component of the reading fluency measure 1 year later. The findings emphasize the contribution of speech rhythm sensitivity in children's reading development, and the authors argue that speech rhythm sensitivity should now be included in current models of children's reading development.
\end{abstract}

Keywords: speech rhythm, prosody, reading, phonological awareness

It is now widely accepted that successful reading development is characterized by more complete phonological representations of words in the mental lexicon, and phonological processing deficits are consistently witnessed in children with reading difficulties (Vellutino \& Fletcher, 2005). Phonological deficits are often accompanied by speech perception deficits (McBride-Chang, 1995), which may compromise the acquisition of phonological codes, interfere with the processing of oral language, and make it more difficult to segment the speech stream into interpretable units such as phonemes and syllables. This is problematic, given that segmental awareness is important for decoding and has been linked to successful reading development (Muter, Hulme, Snowling, \& Taylor, 1998).

There are two types of phonology: Segmental phonology is primarily concerned with separable sound segments in speech, such as phonemes, whereas suprasegmental phonology (prosodic features such as stress, intonation, and timing) relates to overarching patterns or elements of the speech stream. According to Kitzen (2001, p. 42), deficits in speech perception might lead to underspecified representations of both phonemic (segmental) and prosodic (suprasegmental) phonological information, which might result in an underdeveloped system for mapping orthographic information onto phonological representations. However, as Kit-

Andrew J. Holliman and Clare Wood, Faculty of Health and Life Sciences, Coventry University, Coventry, England; Kieron Sheehy, Faculty of Education and Language Studies, Open University, Milton Keynes, England.

The authors gratefully acknowledge the support of the teachers, parents and children who took part in this follow-up study.

Correspondence concerning this article should be addressed to Andrew J. Holliman, Faculty of Health and Life Sciences, Coventry University, Priory Street, Coventry CV1 5FB, England. E-mail: a.holliman@coventry.ac.uk zen noted, although a great deal of research has investigated the role of segmental phonology in children's reading development, the role of suprasegmental phonology is less well understood, and it is speculated that sensitivity to both phonemic and prosodic word structure is necessary for reading proficiency. A literature is now emerging to investigate the role of speech rhythm in reading, and this has led to the development of theoretical models that aim to explain the nature of this relationship on the basis of the available evidence (e.g., Wood, Wade-Woolley, \& Holliman, 2009). The goal of the present study was to investigate the role of speech rhythm sensitivity in a longitudinal study of 5- to 8-yearold English-speaking children.

\section{Speech Rhythm and Word Reading}

According to Kuhn and Stahl (2003), prosodic information is carried by variations in pitch, stress, and duration of utterances, and a growing literature has demonstrated a link between prosodic sensitivity and word reading. For instance, Wood (2006) developed a stress mispronunciations task in which beginning readers were required to listen to a household word that had been mispronounced (where the stress of each word had been reversed) and locate the appropriate picture that corresponded to that word from a line drawing of a house. To successfully complete this task, children need to be sensitive to the stress properties of each word, understand that the word has been incorrectly stressed, and then be able to reverse the stress or apply stress to the unstressed syllable so that the word can be accurately represented and located in the mental lexicon. Indeed, Kitzen (2001) has argued that a reader must be capable of making stress placement shifts in mispronounced words to match the stored lexical code. Wood (2006) found that performance on this task was significantly related to early word reading and spelling. More recently, Holliman, Wood, and Sheehy (2008) found that performance on this task could 
predict a significant amount of unique variance in word reading $(3.8 \%)$ in a group of early readers after controlling for age, vocabulary, phoneme deletion, and rhyme detection ability (phonological awareness). Links between speech rhythm and word reading have also been demonstrated in other recent studies involving stress manipulation and sensitivity (Gutierrez-Palma \& Reyes, 2007; Holliman, Wood, \& Sheehy, 2009, in press; Wood \& Terrell, 1998)

Goswami et al. (2002) investigated whether reading difficulties are associated with deficits in perceptual rhythmic timing. To measure speech rhythm, they used a beat detection task that assessed children's sensitivity to and perception of "rise time," which corresponds to the perceived beat of a spoken syllable. Goswami et al. found that a group of dyslexic children were significantly less sensitive to beat detection than their chronological-age matched counterparts and that performance on this task was able to predict unique variance in word reading $(25 \%)$, spelling $(25 \%)$, and nonword reading (14\%) after controlling for age, nonverbal IQ, and vocabulary. Additionally, after controlling for phonological processing (rhyme oddity) at Step 4, speech rhythm was further able to predict $9 \%$ of the variance in word reading.

To interpret these findings, Goswami et al. (2002) argued that sensitivity to the suprasegmental components of speech might facilitate the development of phonological awareness and reading. More specifically, as beats (peaks in amplitude of the speech signal) corresponded to vowel location, sensitivity to these beats would facilitate the identification of vowels. This, in turn, would enable an individual to locate the onset (the part of the word before the vowel) and rime (the part of the word that includes the vowel and beyond) in words and the boundaries between them, which are important skills in the reading development process (Goswami \& Bryant, 1990). It should be noted that perceptual deficits on measures of beat detection have also been observed in dyslexic adults (Thomson, Fryer, Maltby, \& Goswami, 2006), which suggests that speech rhythm deficits may persist beyond childhood. These studies add weight to the developing argument that speech rhythm is related to word reading.

More recently, Holliman et al. (in press) investigated the relationship between speech rhythm, nonspeech rhythm (rhythmic properties of music or sounds rather than human speech), phonological awareness, and word reading ability in a sample of 102 five- to 7-year-old children. To assess speech rhythm sensitivity, they developed the revised stress mispronunciations task, which required children to recover the correct stress from an incorrectly pronounced word to identify the appropriate graphic that corresponded with that word from a choice of four pictures. Holliman et al. (in press) found that performance on this speech rhythm sensitivity measure was able to account for a significant amount of concurrent variance $(2.1 \%)$ in word reading after controlling for age, vocabulary, phonological awareness, short-term memory, productive nonspeech rhythm, and receptive nonspeech rhythm. The authors argued that although these findings indicate a unique relationship between speech rhythm sensitivity and reading that is not merely subsumed by phonological awareness, less is known about how speech rhythm sensitivity relates to the different components of reading that were not assessed in this study (e.g., reading fluency and reading comprehension) and how it relates to reading development over time.
So how can we explain the observed relationship between speech rhythm and word reading? A variety of possible contributory pathways have been hypothesized in a model outlined by Wood et al. (2009). It has been argued that children are born with a periodicity bias (Cutler \& Mehler, 1993) that allows them to "tune in" to the rhythmic properties of speech in the first language they are exposed to. English, which is a stress-timed language, is characterized by patterns of strong (stressed) and weak syllables, and Cutler and Norris (1988) suggested that sensitivity to such rhythmic properties might facilitate spoken word recognition. Furthermore, English-learning infants appear to be able to segment words on the basis of stress from the age of 7.5 months, to display sensitivity to additional auditory cues that facilitate the identification of word boundaries from 10.5 months, and recognize words from the speech stream at a rate similar to adults by 24 months (Jusczyk, 1999). Wood et al. (2009) anticipated that these word recognition skills facilitate the development of vocabulary (Walley, 1993), phonological awareness, and reading. Such a route to reading ability has been partially supported by Lindfield, Wingfield, and Goodglass (1999), who argued that word-level stress facilitates the perceptual matching process (spoken word recognition), which provides a means for accessing lexical representations, and aids the retrieval of words from the lexicon. In summary, sensitivity to stress (an aspect of speech rhythm) may help infants to segment fluent speech into interpretable units, thus facilitating spoken word recognition, which has been linked to proficient reading (see Metsala, 1997; Wood \& Terrell, 1998); this may also be mediated by vocabulary and phonological awareness.

In addition, Wood (2006) argued in accordance with Chiat (1983) that it is more difficult to decode phonemes in unstressed syllables. Therefore, infants who are more sensitive to stress or who could apply stress to an unstressed syllable should find it easier to recognize the phonemes within words, which would in turn help them to decode words and be able to read them. Such a theory is consistent with Kitzen (2001), who argued that prosodic sensitivity might help to bring some syllables into prominence. For instance, at the word level, prosody can provide reliable cues on the grammatical identity of words (e.g., CONvict and conVICT); at the sentence level, prosody may provide cues to help identify word boundaries. Furthermore, sensitivity to stress may facilitate the categorization of words by rime unit and the identification of onset rhyme boundaries (Goswami, 2003; Goswami et al., 2002). This could enable a child to make analogies between words to decode new ones, a skill that has been linked to reading proficiency (Goswami \& Bryant, 1990).

\section{Speech Rhythm, Reading Fluency, and Reading Comprehension}

Before we investigate the relationship between speech rhythm and reading fluency, we must first discuss what reading fluency is and how it should be measured, because this issue has been much debated in the literature. Typical measures of fluency include a word-per-minute measure (reading rate) and are simply concerned with how fast and accurate a reader is. Many researchers (e.g., Dowhower, 1991; Miller \& Schwanenflugel, 2006, 2008; Schwanenflugel, Hamilton, Kuhn, Wisenbaker, \& Stahl, 2004) have argued that reading with expression is also a key component of reading fluency. Indeed, Kuhn and Stahl (2003) argued that 
given that fluent oral reading is considered to be expressive as well as quick and accurate and that prosodic features are, to a large extent, responsible for such expression, it is important to consider a definition of fluency that encompasses more than rate and accuracy. (p. 18)

This distinction in the literature was acknowledged by Sargent (2004), who investigated the relationship between reading fluency and reading comprehension in 52 children in Grade 5. To assess reading fluency, he used an oral reading fluency measure that essentially measured accuracy and rate, but he also used the Multidimensional Fluency Scale (Zutell \& Rasinski, 1991), which assessed reading fluency by focusing on phrasing and smoothness as well as rate. Sargent found that both fluency measures- that is, the typical measures of fluency (rate and accuracy) and the new fluency measure that incorporated prosodic components (phrasing, smoothness, and pace)—were significantly related to reading comprehension, thus strengthening the association between prosodic reading fluency, typical measures of fluency, and comprehension.

There is a great deal of literature investigating the relationships between prosody, fluency, and comprehension. In an earlier study, Herman (1985) investigated the effect of repeated reading on reading rate, word recognition accuracy, comprehension (measured indirectly), and pausal intrusions during speech (an aspect of prosody), using a sample of eight intermediate-grade students. Herman found that repeated reading enhanced children's reading rate, reading accuracy, and comprehension across passages as expected. However, she also found that repeated reading of a story significantly decreased the number of pausal intrusions within the story, but only within the practiced stories, and thus did not transfer to other stories. Nevertheless, this study demonstrates a link between reading rate and subsequent prosodic awareness.

Related to this, Dowhower (1987) investigated the effect of repeated reading on reading rate, accuracy, comprehension, and prosody in a group of second grade children. Repeated reading was found to improve children's rate, accuracy, comprehension, and prosody; it decreased the number of pausal intrusions (inappropriate hesitations), increased the length of phrases, and improved children's use of intonation (e.g., lowering pitch for final words). Contrary to Herman's (1985) findings, this pattern of improvement was maintained over different passages, not just practiced passages. Dowhower (1987) argued that as children's reading rate, accuracy, and comprehension improves, their prosodic reading improves as well. These findings indicate a positive relationship between aspects of prosody and literacy. However, these studies had some methodological limitations. For instance, the sample sizes in Herman and Dowhower (1987) were eight and 17, respectively, which is very small. Also, both studies used a sample of "less able" children, which perhaps does not adequately inform us about the developmental trajectory of typical readers. Lastly, no published assessment of comprehension was used, so the findings related to these studies should be treated with caution.

In a recent longitudinal study, Miller and Schwanenflugel (2008) investigated the extent to which prosodic sensitivity (as measured at the end of Grade 1 and Grade 2) could predict later reading fluency and reading comprehension in Grade 3. To assess prosody (pausing and intonation), they recorded children's oral reading, converted it to a WAV file, and then scored it using a speech software package. Miller and Schwanenflugel found that children's prosodic reading developed as they progressed through the grades, with a decrease in the number of pausal intrusions leading to more "adult-like" intonation contours. Both pausing and intonation were found to be predictive of word reading skills and also reading comprehension after controlling for word reading. However, pausing at Grade 1 and Grade 2 was unable to predict a significant amount of the variance in fluency at Grade 3 after controlling for word reading, whereas intonation was able to account for a significant amount of the variance in reading fluency after controlling for word reading.

So how does speech rhythm relate to reading fluency and comprehension? Kuhn and Stahl (2003) speculated that prosody might provide a link between reading fluency and reading comprehension. Reading with attention to stress and intonation on particular components of a sentence implies knowledge of the syntactic roles. This is a key component of microprocessing, which helps to arrange the text into hierarchically ordered elements. Readers who are more sensitive to prosodic features while reading make links between speech and reading that might help in the understanding of the text. Kuhn and Stahl (2003) developed this idea further and argued that

appropriate phrasing, intonation, and stress are all considered to be indicators that a child has become a fluent reader .... They act as indicators of the reader's comprehension .... Given that a fluent reader is one that groups text into syntactically appropriate phrases, this parsing of text signifies that the reader has an understanding of what has been read. (p. 6)

The relationship between prosody and reading comprehension has been demonstrated in other studies. For instance, Whalley and Hansen (2006) investigated the relationship between prosody and different components of reading in a group of 81 eight- to 10-yearold children in Grade 4. They found that prosody at the word level predicted unique variance in word reading accuracy, whereas prosody at the phrasal level predicted unique variance in decoding and reading comprehension-both after individual differences in phonological awareness had been controlled. Whalley and Hansen argued that sensitivity to prosodic features such as rhythm and stress facilitate reading comprehension because it enables the individual to segment the speech stream and chunk spoken language into syntactically comprehensible units, which in turn reduce memory load and enable the individual to focus on (comprehend) the more relevant aspects. They also argued that it plays an important role in listening comprehension.

Kitzen (2001) has developed some other theoretical explanations for the observed relationship between prosody and comprehension. For instance, she pointed out that disyllabic nouns are more likely to receive first-syllable stress (e.g., PERmit and CONvict), whereas disyllabic verbs are more likely to receive second-syllable stress (e.g., perMIT and conVICT). Kitzen also noted that compound nouns seem to receive stress on the first element (e.g., BLACKbird and LIGHThouse), whereas noun phrases tend to receive stress on the final element (e.g., black BIRD and light HOUSE). Furthermore, the location of stress within a sentence helps to clarify meaning and point the listener toward the relevant information within a sentence. For example, in the phrase "John kicked the ball," the location of stress changes the meaning of the statement. If the stress falls on "John," it suggests it was he rather than someone else who kicked the ball; if it falls on "kicked," it indicates how he made contact with the ball; and if 
it falls on "ball," it suggests that what he kicked is the most important aspect of the statement. Prosody can also help to indicate whether an utterance is a statement, question, or sarcasm. In summary, prosody seems to help clarify meaning, and this is likely to facilitate comprehension.

Schwanenflugel et al. (2004) proposed two models to account for the relationship between prosody and reading comprehension. In the "reading prosody as partial mediator" model, they argued that proficient, fast, and accurate decoding skills should free up attention resources that can be made available to prosodic processing, which would then have some additional contribution to reading comprehension beyond decoding ability. Thus, prosody might act as a mediator between decoding and reading comprehension. In the "reading comprehension as predictor of reading prosody" model, they argued that children with proficient reading comprehension and decoding ability would be more likely to use prosodic reading.

To inform the legitimacy of these two models, Schwanenflugel et al. (2004) studied how prosody is related to decoding and reading comprehension, using 123 children in Grades 2 and 3 and 24 adults. The prosodic measure converted audio recordings to .WAV files to observe spectrographs that highlighted prosodic features of speech such as pausing and pitch. With regard to the reading prosody as partial mediator model, a strong relationship was found between decoding and prosody, with fluent decoding skills linked to proficient prosody. There was less support for the independent contribution of prosody to comprehension beyond decoding ability. With regard to the reading comprehension as predictor of reading prosody model, a relationship was once again found between decoding and prosody, but not between comprehension and prosody. Schwanenflugel et al. concluded that prosodic reading skills are likely to be evidence that children have proficient decoding ability, but also that prosody and reading comprehension are less related.

It should be noted that the intimate links between fluency and prosody were replicated in a more recent study (Miller \& Schwanenflugel, 2006), using a more sensitive measure of prosody, which also found a stronger link between prosody and comprehension. Interestingly, pitch changes (one aspect of prosody) were able to account for unique variance in reading comprehension after controlling for rapid and accurate text reading; however, pause structures were unable to do so. This finding was supported by Ravid and Mashraki (2007), who also demonstrated stronger links between intonation and comprehension than between pausing and comprehension. This evidence indicates that different aspects of prosody might be related to different aspects of the reading process.

Another explanation for the relationship between prosody and reading has been proposed by Holliman et al. (in press), who argued that stress rules are extremely important while decoding multisyllabic words, due to the variable location of stress depending on the word's suffix. For instance, Wade-Woolley (2007) demonstrated that words ending in -ity or -tion result in a stress shift to the syllable before the suffix of that word, whereas words ending in -ness have stable stress placement. Wade-Woolley argued that children with reading difficulties may be less sensitive to prosodic features of speech, such as stress, and be less able to use morphological rules when decoding words with more than one syllable. In support of this, strong correlations have recently been found between prosody, reading comprehension, and morphological skills (Ravid \& Mashraki, 2007) in a group of 51 Hebrewspeaking children in Grade 4, although Ravid and Mashraki (2007, p. 142) note that there is still a lack of empirical investigation into the relationship between prosody and reading comprehension. This theoretical link was also argued by Wood et al. (2009).

\section{Summary and Rationale}

Although there is a growing literature investigating the relationship between prosody and literacy, there is a distinct lack of longitudinal evidence that investigates whether prosodic sensitivity can predict key components of reading (e.g., word reading, reading fluency, and reading comprehension) over time. In fact, the study by Miller and Schwanenflugel (2008) remains the only longitudinal study of prosodic sensitivity and literacy to date. Therefore, more empirical evidence is required to consolidate recent findings, to see how speech rhythm sensitivity relates to the different components of reading over time, and to do so using different measures of speech rhythm sensitivity. It should be noted that the prosodic measures in Miller and Schwanenflugel (2008) focused on pausing and intonation, so the relationship between stress sensitivity (another aspect of prosody) and the different components of reading over time remains unknown; this signifies the unique contribution of this article.

The purpose of this study is to investigate whether earlier sensitivity to speech rhythm (as measured using the revised stress mispronunciations task from Holliman et al., in press) can predict not only children's vocabulary, rhyme awareness, and phoneme awareness but also their word reading, reading comprehension, and components of reading fluency after controlling for vocabulary and phonological awareness. This study included the key reading measures that have been linked with prosody, along with vocabulary and phonological processing measures to help assess the legitimacy of the model proposed by Wood et al. (2009).

Several key questions were explored in this study to assess the legitimacy of the model proposed by Wood et al. (2009) and to assess some of the theoretical links that have been proposed in the recent literature:

1. Is speech rhythm sensitivity significantly related to measures of phoneme awareness, rhyme awareness, and vocabulary?

2. Is speech rhythm sensitivity significantly related to word reading (1 year later), and does this relationship persist after controlling for age, vocabulary, and phonological awareness?

3. Is speech rhythm sensitivity significantly related to reading comprehension (1 year later), and does this relationship persist after controlling for age, vocabulary, and phonological awareness?

4. Is speech rhythm sensitivity significantly related to the different components of reading fluency - that is, phrasing, smoothness, and pace (1 year later) — and does this relationship persist after controlling for age, vocabulary, and phonological awareness? 


\section{Method}

\section{Participants}

The 102 who participated in Holliman et al. (in press) at Time 1 were invited to participate in this study 1 year later, and the parents of 69 children were successfully contacted and agreed. They were recruited from two combined schools in Buckinghamshire, England, that were comparable in terms of locality, number of students, age range, academic achievement, and the number of pupils with special educational needs. The children's ages ranged between 5 years 11 months and 8 years 8 months (mean age $=7$ years 7 months), and they were in either Year 1, Year 2, or Year 3 classes. All of the men $(n=41)$ and women $(n=28)$ who took part had English as their first language; 11 children had been exposed to a second language within the home. The mean standardized vocabulary score of the sample according to the British Picture Vocabulary Scale II (Dunn, Dunn, Whetton, \& Burley, 1997) was $101.70(S D=10.24)$, which falls in the average score range. The mean word reading raw score according to the British Ability Scales II Word reading subtest (Elliot, Smith, \& McUlloch, $1996)$ was $47.70(S D=18.09)$, which equates to a reading age equivalent of 7 years 10 months.

\section{Procedure}

Information sheets and consent forms were sent out via the school to parents of the 102 children who participated at Time 1. Sixty-eight percent returned their consent forms (69 of 102 parents), and all of these children were willing to take part. Data were collected in May and June 2007, one year after the first phase of data collection, by the primary researcher (Andrew J. Holliman), who was trained to administer the assessment battery. There were eight assessments at Time 2, three of which were also used at Time 1. The assessments were presented in a quasi-randomized order over two sessions to minimize the length of testing period. There were four assessments in each testing session, and these sessions typically lasted approximately $15-20 \mathrm{~min}$.

\section{Time 1 Test Battery}

Vocabulary. Receptive vocabulary was measured using the British Picture Vocabulary Scale II (Dunn et al., 1997). After hearing a word from the administrator, children had to point to the corresponding picture from a choice of four pictures provided. The target words became increasingly difficult and unfamiliar as children progressed through the test, and the test was terminated if children made eight or more errors in any one block of 12 items. Dunn et al. (1997) reported in the British Picture Vocabulary Scale II that Cronbach's alpha reliability coefficient was .94.

Rhyme detection. Rhyme awareness was measured using the rhyme detection subtest of the Phonological Assessment Battery (Frederickson, Frith, \& Reason, 1997). After hearing three words from the administrator, children had to verbally identify the two rhyming words out of the three. Following three practice items there were up to 21 test items (one block of 12 and one block of nine). Children received one point for each correct response, for a maximum score of 21 . The test was terminated if children made eight or more errors in Block 1 and they did not progress onto the second block. This task was also administered at Time 2. It was reported in the Phonological Assessment Battery that Cronbach's alpha reliability coefficient was .92 .

Phoneme deletion. Phoneme awareness was measured using the phoneme deletion task (Wood, 1999). Children had to verbally repeat a word back to the administrator without either the first phoneme (e.g., school would become cool) or the last phoneme (e.g., house would become how). Each subtest had four practice items followed by the 12 test items, for a total score of 24 . This task was also administered at Time 2. Cronbach's alpha reliability coefficient was .94.

The revised mispronunciations task. Speech rhythm sensitivity was measured using the revised mispronunciations task (Holliman et al., in press). Children heard a prerecorded word that was sounded through a speaker, where the stress of that word had been manipulated and reversed. For example, in the normal pronunciation of the word carrot [kærət], the vowel in the first syllable is fully articulated and the vowel in the second syllable is reduced. However, in this task the stress was reversed so that the vowel in the first syllable became reduced and the vowel in the second syllable was fully articulated; carrot was pronounced as "c'rot" [kə'rbt]. To succeed in this task, children would need to be sensitive to the fact that the stress had been manipulated, and be able to recover the correct stress, making a stress shift (Kitzen, 2001) to match the auditory input to a word stored in the lexicon, and then identify the corresponding target item from a choice of four pictures available. The target and distractor items began with the same initial phoneme and were of similar frequency per million, according to the Children's Printed Words Database (http:// www.essex.ac.uk/psychology/cpwd/). The inclusion of distractor items that were matched on word frequency and initial phoneme was an attempt to isolate the speech rhythm (stress manipulation) aspect of the task and to reduce (control for) the potential of vocabulary and phonological awareness during this task. There were a total of 18 mispronounced words, so a score out of 18 was obtained. (See the appendix for the items used in this task.) Cronbach's alpha reliability coefficient was .81 .

\section{Time 2 Test Battery}

Word reading. Word reading ability was measured using the British Ability Scale II Word reading subtest (Elliot et al., 1996). Children read out loud as many words as they could from a list provided, which became increasingly difficult as they progressed through the test. Children received one point for every word read accurately, and the test was terminated if children made eight or more errors in any one block of 10 words. The maximum possible score on this task was 90. This task was also administered at Time 1. It was reported in the British Ability Scale II that Cronbach's alpha reliability coefficient was .98 .

Reading comprehension. Reading comprehension was measured using the revised Neale Analysis of Reading Ability (NARA; Neale, 1997). Following a practice passage, children were required to read up to six passages, depending on the number of errors made (e.g., mispronunciations, substitutions, refusals, additions, omissions, or reversals), as quickly and as accurately as possible. The passages became increasingly difficult to read as children progressed through the test. If 16 or more errors were made in the first five passages or 20 errors in the sixth passage, the 
test was terminated and the scores for that particular passage were not included in the calculations. At the end of each passage in which they had not exceeded the specified number of errors, the children were each asked some open-ended questions about what they had just read. The questions assessed their understanding of the main ideas within the text and the sequence of events, among other aspects, along with some limited inference. There were four comprehension questions for the first passage and eight comprehension questions for the remaining passages, which made a total possible reading comprehension score of 44 . The revised NARA (Neale, 1997) reported that Cronbach's alpha reliability coefficient was .93 .

Reading fluency. Due to the criticisms surrounding the rateper-minute measure of reading fluency, another measurement of fluency was taken that incorporated aspects of expression and prosody. The Multidimensional Fluency Scale, based on Zutell and Rasinski (1991) and used by Sargent (2004), was employed to obtain a fluency score based on phrasing (stress, intonation, expression), smoothness (pauses, hesitations, structure), and pace (slow, fast, conversation speed). In line with the guidance provided with this task, a reading passage was chosen that was well within the range of reading ability in the sample, thus helping to isolate the fluency component of this task. The audio recordings from the first passage of the NARA (Neale, 1997) were chosen for analysis. The primary researcher listened to each audiorecorded passage a number of times and then assigned a score of 1-4 for each category of fluency (phrasing, smoothness, and pace) a higher score indicates more proficient reading fluency.

It should be noted that although the data were collected and scored by the primary researcher (Andrew J. Holliman), efforts were made to ensure that the scorings were reliable and without bias. For instance, the scores obtained from children at Time 1 (1 year previously) were not observed by the primary researcher until all of the assessments at Time 2 had been administered and scored. Thus, the audio recordings on this task were scored before the primary researcher gained access to their scores at Time 1 . The purpose of this was to avoid any influence of prior knowledge about participating children. However, to reduce the potential of observer bias even further, a subsection of audio recordings was also scored by an independent researcher who was not associated with the project but was trained in scoring phrasing, smoothness, and pace on this task. A Pearson correlation addressed the relationship between the ratings of phrasing, smoothness, and pace by the primary researcher and the independent researcher. The correlation between the primary researcher's ratings and the independent researcher's ratings was statistically significant for phrasing $(r=.90, p<.001)$, smoothness $(r=.85, p<.001)$, and pace $(r=$ $.81, p<.001)$, indicating consistent, accurate scoring from the primary researcher. The ratings from the primary researcher were adopted and used in the subsequent analyses.

\section{Results}

Table 1 shows the mean and standard deviation scores on the speech rhythm, reading, and phonological measures taken at Times 1 and 2. It can be seen from Table 1 that participants scored in the upper middle range on the revised mispronunciations task (12.8 from a possible 18) at Time 1. Participants scored in the middle range on the measures of phonological awareness (the phoneme
Table 1

Summary Statistics for Children on the Speech Rhythm, Reading, and Phonological Measures at Time 1 and Time 2

\begin{tabular}{lrr}
\hline \multicolumn{1}{c}{ Task } & $M$ & \multicolumn{1}{c}{$S D$} \\
\hline Time 1 & & \\
Age (in months) & 79.74 & 8.57 \\
Vocabulary (raw scores) & 68.57 & 12.20 \\
Phoneme deletion task (max $=24)$ & 12.62 & 8.01 \\
Rhyme detection task (max $=21)$ & 11.70 & 6.33 \\
BAS Word Reading (max $=90)$ & 31.01 & 20.03 \\
Revised mispronunciations task (max $=18)$ & 12.80 & 3.85 \\
Time 2 & & \\
Phoneme deletion task (max $=24)$ & 16.86 & 6.58 \\
Rhyme detection task (max $=21)$ & 17.13 & 5.65 \\
BAS Word Reading (max $=90)$ & 47.70 & 18.09 \\
Reading comprehension $(\max =44)$ & 10.45 & 5.59 \\
Phrasing (fluency; $\max =4)$ & 3.25 & 0.91 \\
Smoothness (fluency; $\max =4)$ & 3.12 & 0.85 \\
Pace (fluency; max $=4)$ & 3.36 & 0.86 \\
\hline
\end{tabular}

Note. $\quad$ BAS $=$ British Ability Scale

deletion task and the rhyme detection task) at Time 1 and scored in the upper-middle range at Time 2. On the word reading task, participants obtained a higher mean score at Time 2 (47.7) than at Time 1 (31.01). These improvements on the phoneme deletion task, rhyme detection task, and word reading task at Time 2 were expected. Furthermore, relatively high mean scores were obtained on the fluency measures of phrasing, smoothness, and pace (3.25, 3.12 , and 3.36, respectively, from a possible 4).

Table 2 shows the correlation matrix for all the variables included in this study. A Pearson correlation addressed the relationship between the stress sensitivity measure at Time 1 and the battery of reading and phonological awareness assessments at Time 2. An alpha level of .05 was used for all statistical tests.

\section{Is Speech Rhythm Sensitivity Significantly Related to Measures of Phoneme Awareness, Rhyme Awareness, and Vocabulary?}

It can be seen from Table 2 that the revised mispronunciations task at Time 1 was significantly correlated with the measures of phonological awareness at Time 2 (rhyme, $r=.49, p<.001$; phoneme deletion, $r=.48, p<.001$ ). It was also significantly correlated with these phonological awareness measures at Time 1 , along with the measure of vocabulary at Time $1(r=.42, p<$ $.001)$, as expected. Moreover, performance on the revised mispronunciations task at Time 1 was also significantly correlated with word reading at Time $2(r=.63, p<.001)$, reading comprehension at Time $2(r=.61, p<.001)$, and the various components of reading fluency at Time 2 , such as phrasing $(r=.45, p<.001)$, smoothness $(r=.39, p=.001)$, and pace $(r=.43, p<.001)$. The strong relationships found between the revised mispronunciations task and the measures of word reading and comprehension are not surprising, given their documented link in the literature.

The data were inspected to ensure they met the assumptions for a multiple regression analysis. The three fluency components (phrasing, smoothness, and pace) were all negatively skewed. To correct this, we reversed the scores and used a square root transformation. Hierarchical regression analyses were conducted in 
Table 2

Correlation Matrix Between Speech Rhythm, Reading and Phonological Awareness at Time 1 and Time 2

\begin{tabular}{|c|c|c|c|c|c|c|c|c|c|c|c|c|c|}
\hline Variable & 1 & 2 & 3 & 4 & 5 & 6 & 7 & 8 & 9 & 10 & 11 & 12 & 13 \\
\hline 1. T1: Age (in months) & - & & & & & & & & & & & & \\
\hline 2. T1: Vocabulary (raw scores) & $.41^{* * *}$ & - & & & & & & & & & & & \\
\hline 3. T1: Phoneme deletion & $.47^{* * *}$ & $.38^{* *}$ & - & & & & & & & & & & \\
\hline 4. T1: Rhyme detection & .23 & $.56^{* * * *}$ & $.67^{* * * *}$ & - & & & & & & & & & \\
\hline 5. T1: BAS Word Reading & $.58^{* * * *}$ & $.46^{* * * *}$ & $.77^{* * * *}$ & $.67^{* * *}$ & - & & & & & & & & \\
\hline 6. T1: Mispronunciations & $.46^{* * * *}$ & $.42^{* * * *}$ & $.55^{\text {***** }}$ & $.59^{* * * *}$ & $.68^{* * * *}$ & - & & & & & & & \\
\hline 7. T2: Phoneme deletion & $.34^{* *}$ & .22 & $.72^{* * * * *}$ & $.57^{* * * *}$ & $.62^{* * * *}$ & $.48^{* * * *}$ & - & & & & & & \\
\hline 8. T2: Rhyme detection & .18 & $.36^{* *}$ & $.58^{* * * *}$ & $.67^{* * * *}$ & $.58^{* * * *}$ & $.49^{* * * *}$ & $.82^{* * * *}$ & - & & & & & \\
\hline 9. T2: BAS Word Reading & $.42^{* * *}$ & $.35^{* *}$ & $.77^{* * * *}$ & $.69^{* * *}$ & $.9^{* * * * *}$ & $.63^{* * * *}$ & $.67^{* * * *}$ & $.66^{* * *}$ & - & & & & \\
\hline 10. T2: Comprehension & $.38^{* *}$ & $.51^{* * * *}$ & $.68^{* * * *}$ & $.69^{* * *}$ & $.69^{* * *}$ & $.61^{* * * *}$ & $.6^{* * * *}$ & $.58^{* * *}$ & $.75^{* * * *}$ & - & & & \\
\hline 11. T2: Phrasing (fluency) & .19 & $.24^{*}$ & $.4^{* *}$ & $.38^{* *}$ & $47^{* * * *}$ & $.45^{* * *}$ & $41^{* * * *}$ & $.6^{* * * *}$ & $.65^{* * * *}$ & $.5^{* * * *}$ & - & & \\
\hline 12. T2: Smoothness (fluency) & $.32^{* * *}$ & .18 & $.49^{* * * *}$ & $.38^{* *}$ & $.54^{* * * *}$ & $.39^{* *}$ & $.41^{* * * *}$ & $.43^{* * *}$ & $.67^{* * * * *}$ & $.47^{* * * *}$ & $.64^{* * * * *}$ & - & \\
\hline 13. T2: Pace (fluency) & .23 & $.24^{*}$ & $.47^{* * * *}$ & $.4^{* *}$ & $.48^{* * *}$ & $.43^{* * *}$ & $.39^{* *}$ & $.45^{* * *}$ & $.61^{* * * *}$ & $.49^{* * *}$ & $.71^{* * * *}$ & $.81^{* * * * *}$ & - \\
\hline
\end{tabular}

Note. $\quad$ BAS $=$ British Ability Scale.

${ }^{*} p<.05 .{ }^{* * *} p<.01 .{ }^{* * * *} p<.001$.

which age and vocabulary were entered on Steps 1 and 2, respectively, phoneme deletion and rhyme detection entered at Step 3, and speech rhythm sensitivity was entered as the final predictor in the model at Step 4. All of these predictor variables were measured at Time 1 (1 year earlier). It was important to include age in the regressions because age has been shown to be a significant predictor of early literacy attainment. Vocabulary and phonological awareness at Time 1 were included in the regressions to allow a more direct assessment of the model proposed by Wood et al. (2009) and to account for the possible influence of these skills on children's performance on the speech rhythm sensitivity measure when it was assessed (at Time 1). Word reading, reading comprehension, and the reading fluency components (including phrasing, smoothness, and pace) were used as dependent variables in separate analyses (see Table 3 ). All of the criterion variables were measured at Time 2 .

\section{Is Speech Rhythm Sensitivity Significantly Related to Word Reading, and Does This Relationship Persist After Controlling for Age, Vocabulary, and Phonological Awareness?}

It can be seen from Table 3 that after age and vocabulary had been accounted for, phonological awareness at Time 1 was able to account for an additional $45.4 \%$ of the variance in word reading at Time $2, R^{2}$ change $=.454, F(2,64)=43.279, p<.001$. However, speech rhythm sensitivity at Time 1 was able to account for a further $2.2 \%$ of the variance in word reading at Time $2, R^{2}$ change $=.022, F(1,63)=4.360, p=.041$. This indicates that speech rhythm sensitivity can predict unique variance in word reading after controlling for age, vocabulary, and phonological awareness.

\section{Is Speech Rhythm Sensitivity Significantly Related to Reading Comprehension, and Does This Relationship Persist After Controlling for Age, Vocabulary, and Phonological Awareness?}

It can also be seen that after age and vocabulary had been accounted for, phonological awareness at Time 1 was able to account for an additional $29.3 \%$ of the variance in reading comprehension at Time $2, R^{2}$ change $=.293, F(2,64)=$ 22.467, $p<.001$. However, speech rhythm sensitivity at Time 1 was able to account for a further $2.3 \%$ of the variance in reading comprehension at Time $2, R^{2}$ change $=.023, F(1$, $63)=3.747, p=.057$. Although this was not significant, it approached significance.

\section{Is Speech Rhythm Sensitivity Significantly Related to the Different Components of Reading Fluency (Phrasing, Smoothness, and Pace), and Does This Relationship Persist After Controlling for Age, Vocabulary, and Phonological Awareness?}

For the reading fluency measures, although phonological awareness at Time 1 was able to account for an additional $10.8 \%$ of the variance in phrasing at Time 2 after age and vocabulary had been accounted for, $R^{2}$ change $=.108, F(2,64)=4.218, p=.019$, speech rhythm sensitivity at Time 1 was able to account for an additional $5.2 \%$ of variance in phrasing after age, vocabulary, and phonological awareness had been taken into account, $R^{2}$ change $=$ $.052, F(1,63)=4.267, p=.043$.

Moreover, although phonological awareness at Time 1 was able to account for an additional $15.8 \%$ of the variance in smoothness at Time 2 after age and vocabulary had been accounted for, $R^{2}$ change $=.158, F(2,64)=6.883, p=.002$, speech rhythm sensitivity at Time 1 was unable to account for a significant amount of variance in smoothness after age, vocabulary, and phonological awareness had been taken into account, $R^{2}$ change $=$ $.004, F(1,63)=0.328, p=.569$.

Similarly, although phonological awareness at Time 1 was able to account for an additional $15.4 \%$ of the variance in pace at Time 2 after age and vocabulary had been accounted for, $R^{2}$ change $=$ $.154, F(2,64)=6.487, p=.003$, speech rhythm sensitivity at Time 1 was unable to account for a significant amount of variance in pace after age, vocabulary, and phonological awareness had been taken into account, $R^{2}$ change $=.026, F(1,63)=2.260, p=$ .138 
Table 3

Hierarchical Multiple Regression Analysis Predicting Word Reading, Reading Comprehension, Phrasing, Smoothness, and Pace From Age, Vocabulary, Phonological Awareness, and Speech Rhythm Sensitivity

\begin{tabular}{|c|c|c|c|c|}
\hline Step and independent variable & $B$ & $S E B$ & $\beta$ & $\Delta R^{2}$ \\
\hline \multicolumn{5}{|l|}{ 1. Predicting word reading } \\
\hline Age & 0.179 & .194 & .085 & $.173^{* * * * *}$ \\
\hline Vocabulary & -0.175 & .136 & -.118 & .038 \\
\hline Phoneme (PA) & 1.032 & .242 & $.457^{* * * *}$ & $.454^{* * * *}$ \\
\hline Rhyme (PA) & 0.889 & .338 & $.311^{*}$ & \\
\hline Speech Rhythm & 0.947 & .453 & $.201^{*}$ & $.022^{*}$ \\
\hline \multicolumn{5}{|l|}{ 2. Predicting reading comprehension } \\
\hline Age & 0.000 & .067 & .000 & $.141^{* * *}$ \\
\hline Vocabulary & 0.068 & .047 & .148 & $.149^{* * * *}$ \\
\hline Phoneme (PA) & 0.253 & .084 & $.337^{* * *}$ & $.293^{* * * *}$ \\
\hline Rhyme (PA) & 0.226 & .117 & .256 & \\
\hline Speech Rhythm & 0.304 & .157 & .209 & .023 \\
\hline \multicolumn{5}{|l|}{ 3. Predicting phrasing } \\
\hline Age & 0.003 & .005 & .075 & .040 \\
\hline Vocabulary & -0.001 & .004 & -.044 & .031 \\
\hline Phoneme (PA) & -0.009 & .007 & -.234 & $.108^{*}$ \\
\hline Rhyme (PA) & 0.000 & .009 & -.018 & \\
\hline Speech Rhythm & -0.026 & .013 & $-.312^{*}$ & $.052^{*}$ \\
\hline \multicolumn{5}{|l|}{ 4. Predicting smoothness } \\
\hline Age & -0.005 & .005 & -.133 & $.101^{* * *}$ \\
\hline Vocabulary & 0.002 & .003 & .093 & .006 \\
\hline Phoneme (PA) & -0.012 & .006 & -.318 & $.158^{* * *}$ \\
\hline Rhyme (PA) & -0.007 & .009 & -.149 & \\
\hline Speech Rhythm & -0.007 & .011 & -.084 & .004 \\
\hline \multicolumn{5}{|l|}{ 5. Predicting pace } \\
\hline Age & 0.002 & .005 & .047 & .056 \\
\hline Vocabulary & 0.000 & .004 & -.032 & .030 \\
\hline Phoneme (PA) & -0.013 & .006 & -.336 & $.154^{* * *}$ \\
\hline Rhyme (PA) & -0.002 & .009 & -.039 & \\
\hline Speech Rhythm & -0.018 & .012 & -.222 & .026 \\
\hline
\end{tabular}

Note. For phrasing, smoothness, and pace, scores were reversed and square root transformation was used. The regression coefficients (betas) are from the final regression models. $\mathrm{PA}=$ phonological awareness.

${ }^{*} p<.05 .^{* *} p<.01 .^{* * *} p<.001$.

\section{Discussion}

The study set out to examine (a) whether speech rhythm sensitivity was related to children's phonological awareness and vocabulary, and (b) whether speech rhythm sensitivity could predict children's word reading, reading comprehension, and reading fluency (phrasing, smoothness, and pace) 1 year later, after controlling for age, vocabulary, and phonological awareness. These research questions will now be addressed and discussed in turn.

Speech rhythm sensitivity was found to be significantly related to measures of children's phonological awareness (rhyme and phoneme) and vocabulary. We anticipated these strong associations on the basis of the model proposed by Wood et al. (2009). Indeed, children who perform well on this measure of speech rhythm (the revised mispronunciations task) are likely to be more sensitive to stress, have the ability to reverse stress patterns in two-syllable words, and be better able to identify and decode phonemes in words and match these words to those stored in the mental lexicon (Chiat, 1983; Kitzen, 2001; Wood, 2006). Sensitivity to stress may also help children to identify onset rhyme boundaries, which enables and facilitates analogy (Goswami, 2003; Goswami et al., 2002) and may also help to identify word boundaries, facilitating spoken word recognition skills and subse- quent vocabulary development (Kitzen, 2001; Lindfield et al., 1999; Metsala, 1997; Wood \& Terrell, 1998).

Despite the strong associations between speech rhythm, phonological awareness, and vocabulary, a key finding in this study was that performance on the speech rhythm sensitivity measure was able to predict a significant amount of unique variance in word reading and the phrasing component of the reading fluency measure even after individual differences in age, vocabulary, and phonological awareness had been accounted for. However, speech rhythm sensitivity was unable to predict a significant amount of unique variance in reading comprehension after controlling for these variables (although it did approach significance), nor was it able to predict unique variance in the remaining components of the reading fluency measure (smoothness and pace) one year later. The strong association found between speech rhythm sensitivity and word reading in particular was anticipated on the basis of the growing literature that has demonstrated this (Goswami et al., 2002; Gutierrez-Palma \& Reyes, 2007; Holliman et al., 2008, 2009, in press; Miller \& Schwanenflugel, 2006, 2008; Schwanenflugel et al., 2004; Wood, 2006; Wood \& Terrell, 1998). However, it is less clear why speech rhythm sensitivity was not significantly related to reading comprehension or the remaining components of the fluency measure. 
Perhaps the speech rhythm measure in this study, which assessed speech rhythm at the word level, is capturing excess decoding variance that is not accounted for by typical decoding measures. This idea was supported by Kitzen (2001) and Lindfield et al. (1999), who argued that prosodic word structure and wordlevel stress provide a means for accessing lexical representations. This so called "lexical prosody," if considered to be distinct from text-level or sentence-level prosody, might allow one to be a better word decoder in oral reading without necessarily supporting textlevel fluency and subsequent reading comprehension as Kuhn and Stahl (2003) conceived it. Such an explanation is not only consistent with the current findings but also is in line with other research (e.g., Whalley \& Hansen, 2006) that has found word-level prosody to be more strongly related to word reading accuracy (decoding) and phrase-level prosody to be more strongly related to reading comprehension. Furthermore, it is also conceivable that relationships between word-level prosody and reading comprehension, when found, may be explained primarily by the proposed strength of the association between word-level prosody and decoding.

The observed link between speech rhythm at the word level and word reading might be explained via its links with morphology (Holliman et al., in press; Wood et al., 2009). Holliman et al. (in press) argued in line with Holliman et al. (2008) that sensitivity to stress and knowledge of stress rules plays an important role in decoding multisyllabic words; this is because the location of stress in a word varies depending on the word's suffix. Kitzen (2001) and Wade-Woolley (2007) theorized that children with a greater sensitivity to stress (among other prosodic features of speech) may be better able to use these morphological rules when decoding polysyllabic words. However, no measure of morphology was included in this study, so the relationship can only be speculated on here.

The nature of the assessment of prosody (as used in this study) may also help to explain the observed relationship between speech rhythm and the phrasing component of the fluency measure, in the absence of a relationship between speech rhythm and the remaining components (smoothness and pace). The speech rhythm measure in this study essentially assessed children's sensitivity to stress, and of the three components of the Multidimensional Fluency Scale (phrasing, smoothness, and pace), only the phrasing component is assessed on the basis of stress. Therefore, it is perhaps unsurprising that the speech rhythm sensitivity measure was found to be more strongly related to the phrasing component of the fluency measure. Indeed, it would be difficult to score well on the phrasing component of the fluency measure without a good sense of where stress should occur at the word level.

It should also be noted that although speech rhythm sensitivity was unable to predict significant variance in reading comprehension after controlling for age, vocabulary, and phonological awareness, the results were in the expected direction in line with the literature (Kitzen, 2001; Miller \& Schwanenflugel, 2006, 2008; Whalley \& Hansen, 2006) and did approach significance ( $p=$ .057). There are clear links between prosody, fluency, and comprehension in the literature, but the question is, how are these components related together to discriminate between good and poor readers, and how might they explain the strong observed links between speech rhythm sensitivity and reading?

Schwanenflugel et al. (2004) hypothesized that an individual with proficient, fast, and accurate decoding skills should have spare attention resources for prosodic processing. Subsequently, this extra focus on prosodic components may further facilitate comprehension beyond decoding ability. Kuhn and Stahl (2003) have speculated on the way in which prosody may facilitate comprehension, hypothesizing that attention to stress and intonation implies knowledge of syntax, which enables the arrangement of text into hierarchically ordered elements, thus facilitating the comprehension of spoken language. Moreover, Whalley and Hansen (2006) argued that sensitivity to speech rhythm may facilitate the segmentation of words and help the individual to chunk spoken language into syntactically comprehensible units, so that the individual can comprehend the more relevant aspects of the text. It is conceivable that sensitivity to speech rhythm may link (mediate) decoding and reading comprehension.

Although this study offers some unique insights regarding the relationship between prosody (stress) and the different components of reading over time, it does have some limitations. For instance, only one aspect of prosody was measured at Time 1: stress. The speech rhythm measure used in this study was selected because it measures stress, it has good internal reliability, and recent studies have found that performance on this task is significantly related to children's reading development independently of phonological awareness (Holliman et al., 2008 , in press). It was also found to be the best prosodic task (from a selected battery) for discriminating between poor reading and chronological-age and reading-age matched controls (Holliman et al., 2009). However, "it is possible that different aspects of prosody may be linked to different aspects of the reading process" (Miller \& Schwanenflugel, 2008, p. 339). For instance, Miller and Schwanenflugel (2006) found that although pitch changes were able to account for unique variance in reading comprehension, pause structures were not. Ravid and Mashraki (2007) also found stronger links between intonation and comprehension than pausing. Therefore, prosody certainly encompasses other components (e.g., timing, pausing, and tone) that were not explicitly assessed in the prosodic measure included this study, and therefore the findings should perhaps be treated with caution. Further research should consider the ways in which different aspects of prosody are related to the reading process.

In summary, speech rhythm sensitivity (prosody) may play an important role in children's reading development. It might facilitate decoding on different levels (phonemes, rhymes, word recognition, speech perception, and morphology) and may also help bind together decoding, fluency, and reading comprehension, on the basis of evidence from the literature. These findings have practical implications; an interesting possibility is that speech rhythm sensitivity may provide an earlier indication of reading disorder that can be assessed earlier on in childhood, before many phonological awareness measures can be used. As children begin to tune in to the rhythmic properties of speech from birth (Cutler \& Mehler, 1993) and have been shown to be able to segment words on the basis of stress in infancy (Jusczyk, 1999), there is the potential for identification of children at risk of reading disorders very early in development. However, the best way to remediate speech rhythm insensitivity is open to debate; at present there is no speech rhythm intervention study in the literature, although a project of this kind would be timely. 


\section{References}

Chiat, S. (1983). Why Mikey's right and my key's wrong: The significance of stress and word boundaries in a child's output system. Cognition, 14, 275-300.

Cutler, A., \& Mehler, J. (1993). The periodicity bias. Journal of Phonetics, 21, 103-108.

Cutler, A., \& Norris, D. (1988). The role of strong syllables in segmentation for lexical access. Journal of Experimental Psychology: Human Perception and Performance, 14, 113-121.

Dowhower, S. L. (1987). Effects of repeated reading on second-grade transitional readers' fluency and comprehension. Reading Research Quarterly, 22, 389-406.

Dowhower, S. L. (1991). Speaking of prosody: Fluency's unattended bedfellow. Theory Into Practice, 30, 165-175.

Dunn, L. M., Dunn, L. M., Whetton, C., \& Burley, J. (1997). British Picture Vocabulary Scale (2nd ed.). Windsor, England: NFER-Nelson.

Elliot, C. D., Smith, P., \& McUlloch, K. (1996). British Ability Scales (2nd ed.). Windsor, England: NFER-Nelson.

Frederickson, N., Frith, U., \& Reason, R. (1997). Phonological Assessment Battery. Windsor, England: NFER.

Goswami, U. (2003). How to beat dyslexia. The Psychologist, 16, 462465 .

Goswami, U., \& Bryant, P. E. (1990). Phonological skills and learning to read. Hove, England: Erlbaum.

Goswami, U., Thomson, J., Richardson, U., Stainthorp, R., Hughes, D., Rosen, S., \& Scott, S. K. (2002). Amplitude envelope onsets and developmental dyslexia: A new hypothesis. Proceedings of the National Academy of Sciences, USA, 99, 10911-10916.

Gutierrez-Palma, N., \& Reyes, A. P. (2007). Stress sensitivity and reading performance in Spanish: A study with children. Journal of Research in Reading, 30, 157-168.

Herman, P. A. (1985). The effects of repeated readings on reading rate, speech pauses, and word recognition accuracy. Reading Research Quarterly, 20, 553-565.

Holliman, A. J., Wood, C., \& Sheehy, K. (2008). Sensitivity to speech rhythm explains individual differences in reading ability independently of phonological awareness. British Journal of Developmental Psychology, 26, 357-367.

Holliman, A. J., Wood, C., \& Sheehy, K. (2009). A cross-sectional study of prosodic sensitivity and reading difficulties. Manuscript submitted for publication.

Holliman, A. J., Wood, C., \& Sheehy, K. (in press). The contribution of sensitivity to speech rhythm and nonspeech rhythm to early reading development. Educational Psychology.

Jusczyk, P. W. (1999). How infants begin to extract words from speech. Trends in Cognitive Sciences, 3, 323-328.

Kitzen, K. (2001). Prosodic sensitivity, morphological ability, and reading ability in young adults with and without childhood histories of reading difficulty (Unpublished doctoral dissertation). Columbia University, New York, NY.

Kuhn, M. R., \& Stahl, S. A. (2003). Fluency: A review of developmental and remedial practices. Journal of Educational Psychology, 95, 3-21.

Lindfield, K., Wingfield, A., \& Goodglass, H. (1999). The role of prosody in the mental lexicon. Brain and Language, 68, 312-317.

McBride-Chang, C. (1995). Phonological processing, speech perception, and reading disability: An integrative review. Educational Psychologist, $30,109-121$.

Metsala, J. L. (1997). Spoken word recognition in reading disabled children. Journal of Educational Psychology, 89, 159-169.

Miller, J., \& Schwanenflugel, P. J. (2006). Prosody of syntactically complex sentences in the oral reading of young children. Journal of Educational Psychology, 98, 839-853.

Miller, J., \& Schwanenflugel, P. J. (2008). A longitudinal study of the development of reading prosody as a dimension of oral reading fluency in early elementary school children. Reading Research Quarterly, 43, $336-354$.

Muter, V., Hulme, C., Snowling, M., \& Taylor, S. (1998). Segmentation, not rhyming, predicts early progress in learning to read. Journal of Experimental Child Psychology, 71, 3-27.

Neale, M. (1997). The Neale Analysis of Reading Ability II. Windsor, England: NFER-Nelson.

Ravid, D., \& Mashraki, Y. E. (2007). Prosodic reading, reading comprehension and morphological skills in Hebrew-speaking fourth graders. Journal of Research in Reading, 30, 140-156.

Sargent, S. E. (2004). Oral reading fluency: A predictor of reading achievement. Balanced Reading Instruction, 11, 1-16.

Schwanenflugel, P. J., Hamilton, A. M., Kuhn, M. R., Wisenbaker, J. M., \& Stahl, S. A. (2004). Becoming a fluent reader: Reading skill and prosodic features in the oral reading of young readers. Journal of Educational Psychology, 96, 119-129.

Thomson, J. M., Fryer, B., Maltby, J., \& Goswami, U. (2006). Auditory and motor rhythm awareness in adults with dyslexia. Journal of Research in Reading, 29, 334-344.

Vellutino, F. R., \& Fletcher, J. M. (2005). Developmental dyslexia. In M. J. Snowling \& C. Hume (Eds.), The science of reading: A handbook (pp. 362-378). Oxford, England: Blackwell.

Wade-Woolley, L. (2007, July). Stress assignment and derivational morphology in skilled reading. Paper presented at the annual meeting of the Society for the Scientific Study of Reading, Prague, Czech Republic.

Walley, A. C. (1993). The role of vocabulary development in children's spoken word recognition and segmentation ability. Developmental Review, 13, 286-350.

Whalley, K., \& Hansen, J. (2006). The role of prosodic sensitivity in children's reading development. Journal of Research in Reading, 29, 288-303.

Wood, C. (1999). The contribution of analogical problem solving and phonemic awareness to children's ability to make orthographic analogies when reading. Educational Psychology, 19, 277-286.

Wood, C. (2006). Metrical stress sensitivity in young children and its relationship to phonological awareness and reading. Journal of Research in Reading, 29, 270-287.

Wood, C., \& Terrell, C. (1998). Poor readers' ability to detect speech rhythm and perceive rapid speech. British Journal of Developmental Psychology, 16, 397-413.

Wood, C., Wade-Woolley, L., \& Holliman, A. J. (2009). Phonological awareness: Beyond phonemes. In C. Wood \& V. Connelly (Eds.), Contemporary perspectives on reading and spelling (pp. 7-23). London, England: Routledge.

Zutell, J., \& Rasinski, T. V. (1991). Training teachers to attend to their students' oral reading fluency. Theory Into Practice, 30, 211-217. 


\section{Appendix A}

\section{Phonetic Transcription and Word Frequency per Million for All Target and Distractor} Items

\begin{tabular}{|c|c|c|c|c|c|}
\hline \multirow{2}{*}{$\begin{array}{l}\text { Target words } \\
\text { (frequency) }\end{array}$} & \multirow{2}{*}{$\begin{array}{c}\text { Phonetic } \\
\text { transcription }\end{array}$} & \multirow{2}{*}{$\begin{array}{l}\text { Stress reverse } \\
\text { condition }\end{array}$} & \multicolumn{3}{|c|}{ Distractor items (frequency) } \\
\hline & & & Item 1 & Item 2 & Item 3 \\
\hline spider (93) & 'spaIdə & spə'd3: & swinging (83) & snowman (62) & sandwich (83) \\
\hline baker (93) & 'beIkə & be'Ik3: & beetles (83) & branches (93) & bottles (93) \\
\hline barrel (10) & 'bærəl & bə'rel & bracelet (10) & burglars (10) & ballet (10) \\
\hline builder (21) & 'bIldə & bəl'd3: & blackbird (31) & biscuit (21) & bookcase (21) \\
\hline butcher (41) & 'butfa & bə't $\int 3:$ & baseball (52) & badgers (31) & boiling (52) \\
\hline butter (175) & 'bıtว & bə't3: & breakfast (196) & bottle (186) & basket (186) \\
\hline carrot (21) & 'kærət & kə'rdt & clipboard (10) & cutting (10) & camel (21) \\
\hline cleaner (83) & 'kli:nə & klə'n3: & crying (72) & counting (62) & cupboard (93) \\
\hline cooker (31) & 'kukə & kə'k3: & carrots $(31)$ & cowboy (31) & crayons (31) \\
\hline jumper (114) & 'd $3 \wedge \mathrm{mp} ә$ & dzəm'pз: & jewels (114) & jolly (103) & jacket (93) \\
\hline mirror (41) & 'mIrə & mə'ro: & married (41) & mushrooms (31) & marbles (52) \\
\hline painter (21) & 'pentə & pən't3: & panda (31) & penguin $(21)$ & peanuts (21) \\
\hline parrot (83) & 'pærət & po'rot & pattern (72) & pumpkin (62) & pocket (62) \\
\hline plaster (52) & 'pla:stə & pləs't3: & pencil (52) & penny (41) & pizza (41) \\
\hline rubber (10) & rs bə & rə'b3: & rhino (31) & raining (10) & robot (21) \\
\hline ruler (10) & 'ru:lə & rə'l3: & rowing (10) & robin $(31)$ & rainbow (21) \\
\hline sailor (10) & 'seIlə & sə'lo: & swimmer (10) & smiling (10) & scarecrow (21) \\
\hline singer (10) & 'sInə & səy'3: & swordfish (10) & skateboard (10) & seagull (10) \\
\hline tiger (52) & 'taIgə & to'g3: & tissue $(31)$ & tractor $(31)$ & twenty (31) \\
\hline
\end{tabular}

Note. The word frequencies in parentheses are per million.

Received September 10, 2008 